\title{
Gibbs-Donnan Potential as a Tool for Membrane Vesicles Polarization
}

\author{
Mazur Iuliia, Kosterin Sergiy, Veklich Tetyana, Shkrabak Oleksandr \\ Palladin Institute of Biochemistry, National Academy of Sciences of Ukraine, Kiev, Ukraine \\ Email: yuliya.vorona@gmail.com
}

Received 28 April 2014; revised 12 May 2014; accepted 19 May 2014

Copyright (C) 2014 by authors and Scientific Research Publishing Inc.

This work is licensed under the Creative Commons Attribution International License (CC BY).

http://creativecommons.org/licenses/by/4.0/

(c) (i)

\section{Abstract}

It has been theoretically predicted that under conditions leading to Gibbs-Donnan equilibrium in case when size of one compartment is very different from another (as in system "membrane vesicle/liposomes-incubation medium") stable transmembrane potential can be formed, which value is sufficient to fit requirement of real transmembrane potential. Four partial cases were considered with different location and charge of impermeable ion and it was concluded that locations of impermeable ions in medium provide stable transmembrane potential with sufficient value of $60-70 \mathrm{mV}$. Potential-sensitive probe, such as DiOC $_{6}(3)$ and oxonol VI, were used to confirm the calculated potential. According to the change in fluorescence level and emission/excitation shift, a stable and relatively high transmembrane potential can be formed if salt of impermeable ion is located in incubation medium. Impermeable cations and anions may be used to create positive and negative transmembrane potential respectively.

\section{Keywords}

Gibbs-Donnan Effect, Membrane Vesicles, Membrane Polarization

\section{Introduction}

Membrane potential is a usual characteristic of all the living cells. It is created by ion gradient across the lipid bilayer and reaches values in range $-90 \ldots-50 \mathrm{mV}$ in the state of rest [1]-[4] depending on the cell type. Due to changes in magnitude of membrane potential, cells are able to transmit nerve pulses [5] [6], couple excitation-contraction [7], communicate [8], proliferate [9], and fertilize [10]. Goldman-Hodgkin-Katz voltage equation can be used to calculate value of transmembrane potential (1):

$$
V=\frac{R T}{F} \ln \left(\frac{P_{\mathrm{Na}}\left[\mathrm{Na}^{+}\right]_{\text {out }}+P_{K}\left[\mathrm{~K}^{+}\right]_{\text {out }}+P_{\mathrm{Cl}}\left[\mathrm{Cl}^{+}\right]_{\text {out }}}{P_{\mathrm{Na}}\left[\mathrm{Na}^{+}\right]_{\text {in }}+P_{K}\left[\mathrm{~K}^{+}\right]_{\mathrm{in}}+P_{\mathrm{Cl}}\left[\mathrm{Cl}^{+}\right]_{\text {in }}}\right),
$$


A simplified formula is used if the only one ion is permeable ( $\mathrm{K}^{+}$for example) (2):

$$
V=\frac{R T}{F} \ln \left(\frac{\left[K^{+}\right]_{\text {out }}}{\left[K^{+}\right]_{\text {in }}}\right),
$$

Change of membrane potential value can lead to changes in properties of transmembrane proteins (e.g., change in mobility of membrane component affects protein function [11]) both in eukaryotic and prokaryotic cells [12] [13]. The most popular methods of using membrane vesicle include determining properties of membrane protein, characterization of transport system [14]-[21], study of substrate transport and transporter drug binding kinetics, assign phase behavior of lipid mixtures in bilayers [22] [23]. It should be emphasized that all these processes are sensible to changes of membrane potential. Especially membrane transport and its kinetic parameters, e.g., rates of reactions involving translocation of charged species across the membrane [24] and binding to the membrane [25], are influenced by membrane potential. In majority of works, polarization of membrane vesicles is achieved by ion gradients in the presence of ionophores as valinomycin-generated $\mathrm{K}^{+}$diffusion potential [14] [16]-[20]. Such methods allow creation of unstable potential with decreasing or dissipating value. Thus, using potassium-valinomycin system provides sufficient but unstable potential that fully disappears in 3 - 5 minutes [26].

The aim of this work was to find a tool for creating stable and sufficient value of transmembrane potential in membrane vesicles.

\section{Methods}

Plasma membrane vesicles of smooth muscle cell were prepared from swine myometrium as was described previously [27]. Finally, membrane fraction was resuspended in medium that contain $300 \mathrm{mM} \mathrm{KCl}$.

Protein quantity content in membrane fraction was determined by Bradford method [28].

Transmembrane potential was measured by potential-sensitive fluorescent dyes and all fluorescent experiments were carried out in PTI spectrophotometer. To measure positive-inside membrane potential potentialsensitive dye $\operatorname{DiOC}_{6}(3)$ (3,3'-Dihexyloxacarbocyanine Iodide) with final concentration $2.5 \mu \mathrm{M}$ was used. The excitation wavelength was set to $452 \mathrm{~nm}$ (slit width $5 \mathrm{~nm}$ ) and emission to $506 \mathrm{~nm}$ (slit width $5 \mathrm{~nm}$ ). After the fluorescence signal was constant the aliquot of plasma membrane vesicle $(66 \mu \mathrm{g})$ was added. Fluorescence changes was measured as coefficient $\tau=F_{v} / F_{\text {base }}$, where $F_{\text {base }}$-fluorescence signal prior to membrane vesicle addition, $F_{v}$-florescence signal after membrane vesicle addition.

To measure negative-inside membrane spectrum shift of potential-sensitive dye oxonol VI was used. It final concentration was $1 \mu \mathrm{M}$, protein content was $200 \mu \mathrm{g} / \mathrm{ml}$.

Results were analyzed by Wilcoxon signed-rank test, $\mathrm{p}<0.01$ (MS Excel).

Reagents used were as follows: $\operatorname{DiOC}_{6}(3)$, Hepes, sucrose (Sigma, USA); Tris-hydroxymethyl-aminomethane (Reanal, Hungary); oxonol VI (Fluka, Switzerland). Other reagents were of domestic production of analytical and chemical purity.

\section{Results}

\subsection{Calculations of Gibbs-Donnan Transmembrane Potential on Membrane Vesicle}

Gibbs-Donnan equilibrium [29] is conceived when there are two compartments separated by half-permeable membrane and there are impermeable ions in one of the compartments and permeable salt in both ones. But obtained value of Donnan potential is not high, only about $\pm 11 \mathrm{mV}$. However, earlier Prof. Kosterin predicted [30] that Donnan effect can be used for creation of stable, equilibrium membrane potential if compartments volumes significantly differ (3).

$$
w_{e} \gg w_{i},
$$

where $w_{e}$ and $w_{i}$ summary volume of each compartments. This work continues his studies.

In calculations electrochemical system contains two compartments which divided by half-permeable membrane. In one of the compartment there permeable salt, which is potassium chloride $(b)-\mathrm{KCl}$, because it is one of the most ubiquitous ion in organism. At the beginning of our study we did not know what is better to use: salt of impermeable anion with potassium $(x)$ - KA, or cation with chloride $(z)-\mathrm{CatCl}$, and where it is better to be 
placed-inside (lower index $i$ ) or outside the vesicle (lower index $e$ ). So general formula and four special cases (Figure 1) of it was reviewed:

A) Impermeable anion inside vesicles.

B) Impermeable cation inside vesicles.

C) Impermeable anion outside vesicles.

D) Impermeable cation outside vesicles.

In calculation it is important to maintain condition for initial isotonicity (4):

$$
b_{0, i}+z_{0, i}+x_{0, i}=b_{0, e}+z_{0, e}+x_{0, e}
$$

where $b_{0, i}$-initial concentration of $\mathrm{KCl}$ inside vesicles, $z_{0, i}$-initial concentration of CatCl inside vesicles, $x_{0, i}-$ initial concentration of $\mathrm{KA}$ inside vesicles, $b_{0, e}$-initial concentration of $\mathrm{KCl}$ in incubation medium, $z_{0, e}$ - initial concentration of CatCl in incubation medium, $x_{0, e}$-initial concentration of KA in incubation medium. According to this sum over all the salts concentrations must be the same in two compartments. This principle is involved by the fact that osmotic power in two compartments should be equal.

To diverse general formula of Gibbs-Donnan potential several principles are used as input data.

1) Particles balance (5) (6). It describes particles balance before and after equilibrium between incubation medium $\left(w_{e}\right)$ and vesicles $\left(w_{i}\right)$ and considering equality of salt and ions concentration.

$$
\begin{aligned}
& b_{0, e} w_{e}+x_{0, e} w_{e}+b_{0, i} w_{i}+x_{0, i} w_{i}=\left[K_{e q}^{+}\right]_{e} w_{e}+\left[K_{e q}^{+}\right]_{i} w_{i}, \\
& b_{0, e} w_{e}+z_{0, e} w_{e}+b_{0, i} w_{i}+z_{0, i} w_{i}=\left[C l_{e q}^{-}\right]_{e} w_{e}+\left[C l_{e q}^{-}\right]_{i} w_{i},
\end{aligned}
$$

where $\left[K_{e q}^{+}\right]_{e},\left[K_{e q}^{+}\right]_{i}$-concentration of potassium ions in equilibrium point in incubation medium and vesicles correspondingly, $\left[\mathrm{Cl}_{e q}^{-}\right]_{e},\left[\mathrm{Cl}_{e q}^{-}\right]_{i}$-concentration of chloride ions in equilibrium point in incubation medium and vesicles correspondingly.

2) Equality of electrochemical potentials $(\mu)$ in Equilibrium (7)-(12). Electrochemical potential created by every ion inside of vesicle is equal to potential formed on the external side of membrane.

$$
\begin{gathered}
\mu_{K_{i}^{+}}=\mu_{K_{0}^{+}}+R T \ln \left[K_{e q}^{+}\right]_{i}+F V_{i}, \\
\mu_{K_{e}^{+}}=\mu_{K_{0}^{+}}+R T \ln \left[K_{e q}^{+}\right]_{e}+F V_{e}, \\
\mu_{C l_{i}^{-}}=\mu_{C l_{0}^{-}}+R T \ln \left[C l_{e q}^{-}\right]_{i}-F V_{i}, \\
\mu_{C l_{e}^{-}}=\mu_{C l_{0}^{-}}+R T \ln \left[C l_{e q}^{-}\right]_{e}-F V_{e}, \\
\mu_{K_{i}^{+}}=\mu_{K_{e}^{+}}, \\
\mu_{C_{i}^{-}}=\mu_{C l_{e}^{-}},
\end{gathered}
$$

3) Electrical neutrality (13) (14), which describes balance of positive and negative charge in each side of membrane:

$$
\begin{aligned}
& {\left[\mathrm{K}_{e q}^{+}\right]_{i}+\mathrm{z}_{0, i}=\left[\mathrm{Cl}_{e q}^{-}\right]_{i}+x_{0, i},} \\
& {\left[\mathrm{~K}_{e q}^{+}\right]_{e}+z_{0, e}=\left[\mathrm{Cl}_{e q}^{-}\right]_{e}+x_{0, e}}
\end{aligned}
$$

Considering three principles mentioned above (4)-(14) and the calculation transmembrane potential equation (2), taking into account compartments size, a general formula for calculating transmembrane potentials caused by Gibbs-Donnan effect was obtained (14).

$$
\begin{aligned}
E_{D}= & \frac{R T}{F} \ln \frac{1}{2\left(b_{0, e}+x_{0, e}+\theta \cdot b_{0, i}+\theta \cdot z_{0, i}\right)}\left(x_{0, i}+\theta \cdot z_{0, e}-z_{0, i}-\theta \cdot x_{0, e}\right. \\
& \left.+\sqrt{\left(z_{0, i}-x_{0, i}+\theta \cdot z_{0, e}+\theta \cdot x_{0, e}\right)^{2}+4\left(b_{0, e}+x_{0, e}+\theta \cdot b_{0, i}+\theta \cdot z_{0, i}\right)\left(b_{0, e}+\theta \cdot b_{0, i}+\theta \cdot x_{0, i}+z_{0, e}\right)}\right)
\end{aligned},
$$


where $\theta=\frac{w_{i}}{w_{e}}$.

As it was predicted by Kosterin [30], the higher difference between compartment volumes is, the higher Donnan potential can emerge. To validate this assumption, dependence of transmembrane potential on value of $\theta$ was visualized (Figure 2).

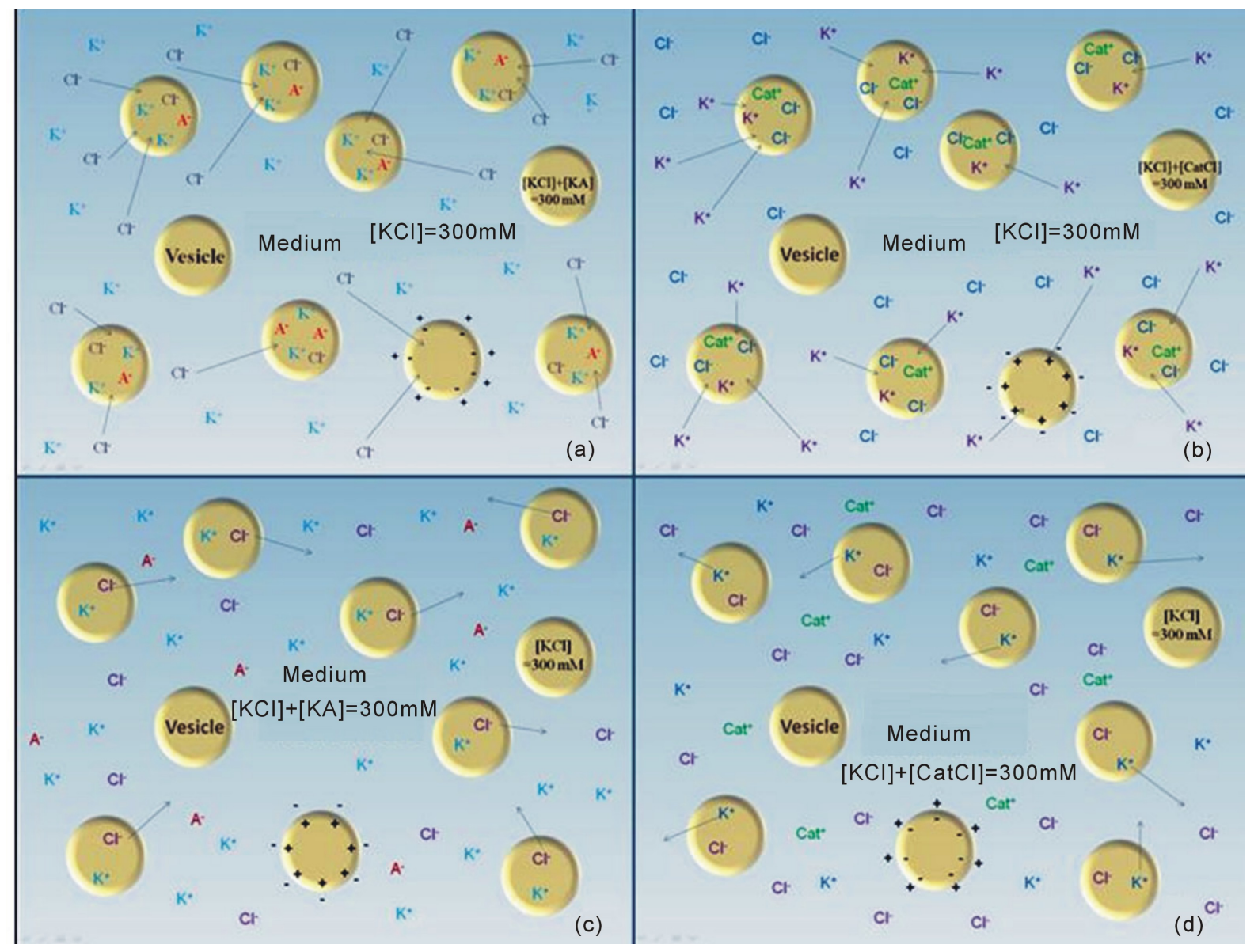

Figure 1. Gibbs-Donnan effect in polarization of membrane vesicle. There are four cases differing in impermeable ion charge and location. (a) There is potassium chloride in the medium and there are potassium chloride and salt of potassium with impermeable anion inside the vesicle. Concentrations of potassium chloride in the medium and sum of potassium chloride and potassium anion concentrations in vesicle are equal, in other words $[\mathrm{KCl}]_{I}=[\mathrm{KCl}]_{e}+[\mathrm{KA}]_{e}$. Since concentration of chloride ions in medium is higher, due to the concentration gradient chloride ions move into the vesicle. At the same time concentration of impermeable anions inside vesicle is higher than in medium, but membrane is not permeable for it, so anions cannot move outside vesicle. Taking all the points into account additional negative charge inside the vesicles is created. (b) There is potassium chloride in medium and there are potassium chloride and salt of impermeable cation with chloride inside vesicle. Concentrations of potassium chloride in medium and sum of potassium chloride and cation chloride concentrations in vesicle are equal, that means $[\mathrm{KCl}]_{I}=[\mathrm{KCl}]_{e}+[\mathrm{CatCl}]_{e}$. Due to the concentration gradient potassium ions move into the vesicle. Like in (a) concentration of impermeable ion inside vesicle is higher than in medium, but membrane is not permeable for it, so cations stay inside. Thus, additional positive charge inside the vesicles is created. (c) There is potassium chloride in vesicles and there are potassium chloride and salt of impermeable anion with potassium in medium. Concentrations of potassium chloride in vesicles and sum of potassium chloride and potassium anion concentrations in medium are equal, so $[\mathrm{KCl}]_{I}+[\mathrm{KA}]_{I}=[\mathrm{KCl}]_{e}$. Due to the concentration gradient chloride ions move out from the vesicles, and impermeable anions stay in medium because membrane is not permeable for them. Additional negative charge outside the vesicles is created. (d) There is potassium chloride in vesicles and there are potassium chloride and salt of impermeable cation with chloride concentrations in medium. Salts concentration must fit the following equation: $[\mathrm{KCl}]_{I}+[\mathrm{CatCl}]_{I}=$ $[\mathrm{KCl}]_{e}$. Due to the concentration gradient potassium ions move out from the vesicles, and impermeable cations stay in medium because membrane is not permeable for them. Additional positive charge outside the vesicles is created. 


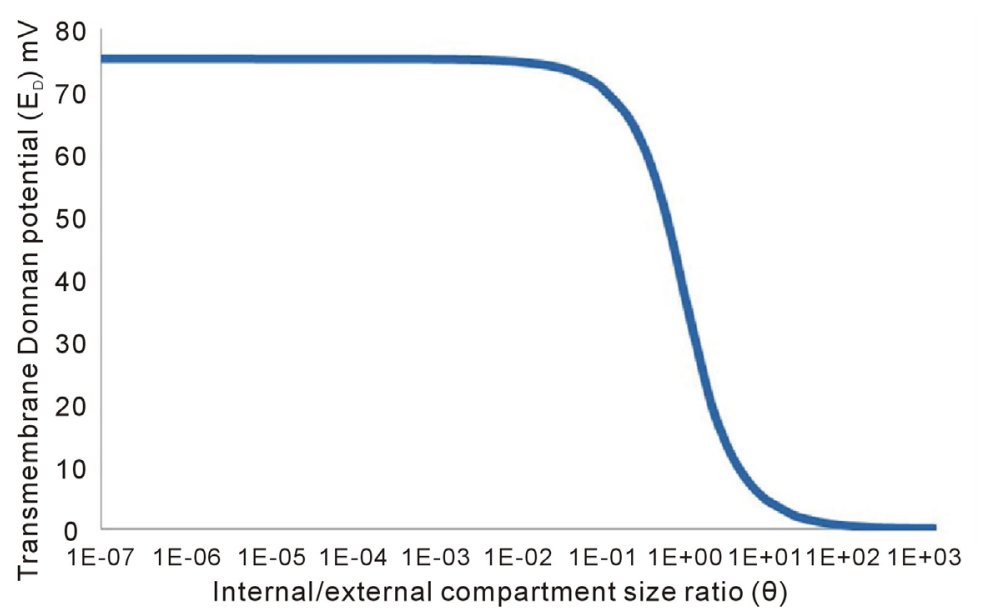

Figure 2. Theoretically calculated increase of transmembrane Donnan potential depending on internal/external compartment size ratio $(\theta)$. Some random salt concentrations were used.

From the Figure 2, it is clear that the lower $\theta$ is, the higher Donnan potential can be formed. It is known that size of vesicle is quite small, about $0.3-0.4 \mu \mathrm{m}$, which is confirmed in our laboratory by electron microscopy and hydrodynamic diameter measurement (data is not shown). Medium volume is two or three order of magnitude as big as the sum over all the vesicle volumes, which means that volumes of compartments significantly differ as (3), thus, $\theta \rightarrow 0$, so that expression 15 is simplified to (16):

$$
\lim _{\theta \rightarrow 0} E_{D}=\frac{R T}{F} \ln \left(\frac{x_{0, i}-z_{0, i}+\sqrt{\left(z_{0, i}-x_{0, i}\right)^{2}+4\left(b_{0, e}+x_{0, e}\right)\left(b_{0, e}+z_{0, e}\right)}}{2\left(b_{0, e}+x_{0, e}\right)}\right) .
$$

It was mentioned that there are four cases depending on location and charge of impermeable ion. First one is when anions are located inside vesicle, in other words, in smaller compartment (Figure 1(a)). Due to the Donnan effect negative charge appears inside vesicle. We considered it as a positive transmembrane potential, in other words, transmembrane potential is positive if internal side of membrane has bigger negative charge than external side has.

So when $x_{0, e}, z_{0, e}, z_{0, i}=0$, the value of Donnan potential can be obtained from the simplified Equations (16).

$$
E_{D}=\frac{R T}{F} \ln \left(\frac{x_{0, i}+\sqrt{x_{0, i}^{2}+4 b_{0, e}^{2}}}{2 b_{0, e}}\right) .
$$

So dependence of membrane potential on impermeable anion concentration was obtained (Figure 3).

Figure 3 shows that the higher concentration of impermeable salt is used the higher Donnan potential can emerge. Nevertheless value of it is insufficient for further investigation, because value of live cells transmembrane potential is 6 times as big as the value that may be obtained. In real experimental conditions overall salt concentration higher than $0.3 \mathrm{mM}$ cannot be used because it is far from real osmotic power in the tissue or cell. Such a result is quite similar to what appears in the second case, whereas salt of impermeable cation is located inside vesicle (data is not shown) and $x_{0, i}, x_{0, e}, z_{0, e}=0$. There is only one difference-the charge inside the vesicles is opposite. Hence location of impermeable ions regardless to its charge cannot provide sufficient value of transmembrane potential. Yet there are two others cases, where impermeable ions are located in medium, in other words in a compartment which volume is much higher.

In third case, impermeable anions are located in medium, outside the vesicles (Figure 1(c)). The value of Donnan potential in conditions when $x_{0, i}, z_{0, i}, z_{0, e}=0$ can be obtained from a quite simple formula (18):

$$
E_{D}=\frac{R T}{F} \ln \sqrt{\frac{b_{0, e}}{b_{0, i}}} .
$$




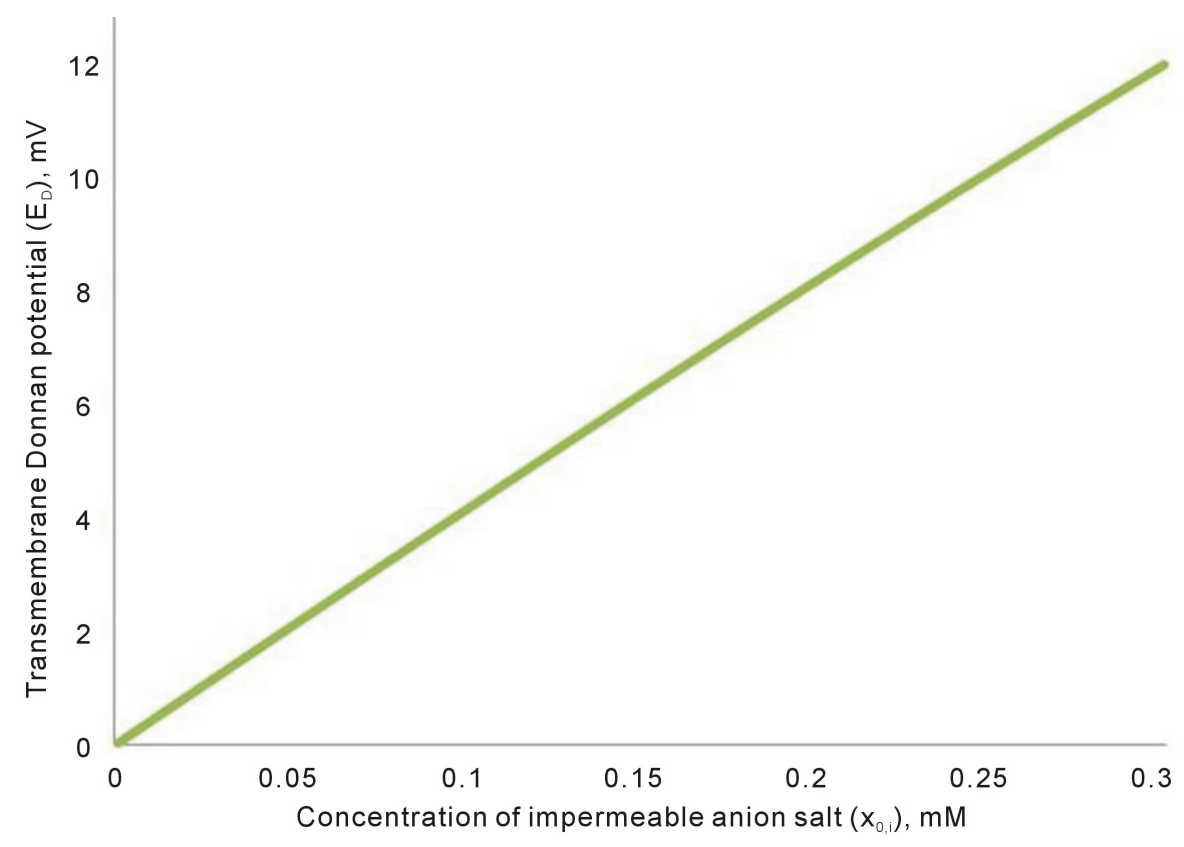

Figure 3. Calculations of influence of concentration salt of impermeable anion on the value of Donnan potential $\left(E_{D}\right)$ in case when initially salt of impermeable anion $\left(x_{0, i}\right)$ is located inside vesicle. Salts concentrations satisfy such demand $x_{0, i}+b_{0, i}=b_{0, e}=300 \mathrm{mM}$.

Dependence of transmembrane potential on impermeable salt is like in the previous cases, the higher concentration is, the higher potential is. However, value of Donnan potential is higher comparing to cases where impermeable ions are inside vesicle (Figure 4). Its value fits range observed in real cells, thus, it can be used as a tool in experiments for membrane polarization.

Both results with impermeable ions inside vesicles and results with ions in medium are quite similar (Figure 5) and have one difference-charge of polarization. In four case dependence on salt concentration in conditions when $\mathrm{x}_{0, e}, x_{0, i}, z_{0, i}=0$ also resembles previous (19):

$$
E_{D}=\frac{R T}{F} \sqrt{\frac{b_{0 . i}}{b_{0, e}}} .
$$

\subsection{Positive Potential on Vesicle Is Proven by Changing of $\mathrm{DiOC}_{6}(3)$ Fluorescence Level}

This dye was used for checking the polarization existence in case when in medium there is impermeable cation salt present. Due to our calculations negative charge should be accumulated inside vesicle (Figure 1(d), Figure 5). According to literature data the fluorescent signal of $\mathrm{DiOC}_{6}(3)$ is drop if hyperpolarization occur and rise during depolarization [31], thus in this case of vesicle polarization fluorescence signal should decrease.

As a control experiment the medium which contained only $300 \mathrm{mM} \mathrm{KCl}$ was used. In a sample probe medium contained $297 \mathrm{mM}$ cholin chloride and $3 \mathrm{mM} \mathrm{KCl}$ (as some amount of it added with membrane vesicle aliquot). According to our calculation in such conditions transmembrane potential about $-65 \mathrm{mV}$ should appear.

When dye added to medium there was an initial period of slow decline in light emission. Vesicle was not added until a steady level was reached (about 5 minute). After vesicle addition rapid signal increase was observed, which level was stable during at least 10 minutes. Finally, SDS $0.005 \%$ was used and both signal as in control, so in sample reached one level of florescence (Figure 6).

Thus, Gibbs-Donnan potential is stable in time. Additional experiments show that equilibrium potential is stable during at least 10 minutes.

To compare fluorescent signal in probe and control often base level of dye fluorescence was differ, so coefficient $\tau$ was used ( $\tau=F_{v} / F_{\text {base, }}$, where $F_{\text {base }}$-fluorescence signal prior to membrane vesicle addition, $F_{v}-$ fluorescence signal after membrane vesicle addition). In Figure 7, there is comparison of average meaning of $\tau$ in con- 


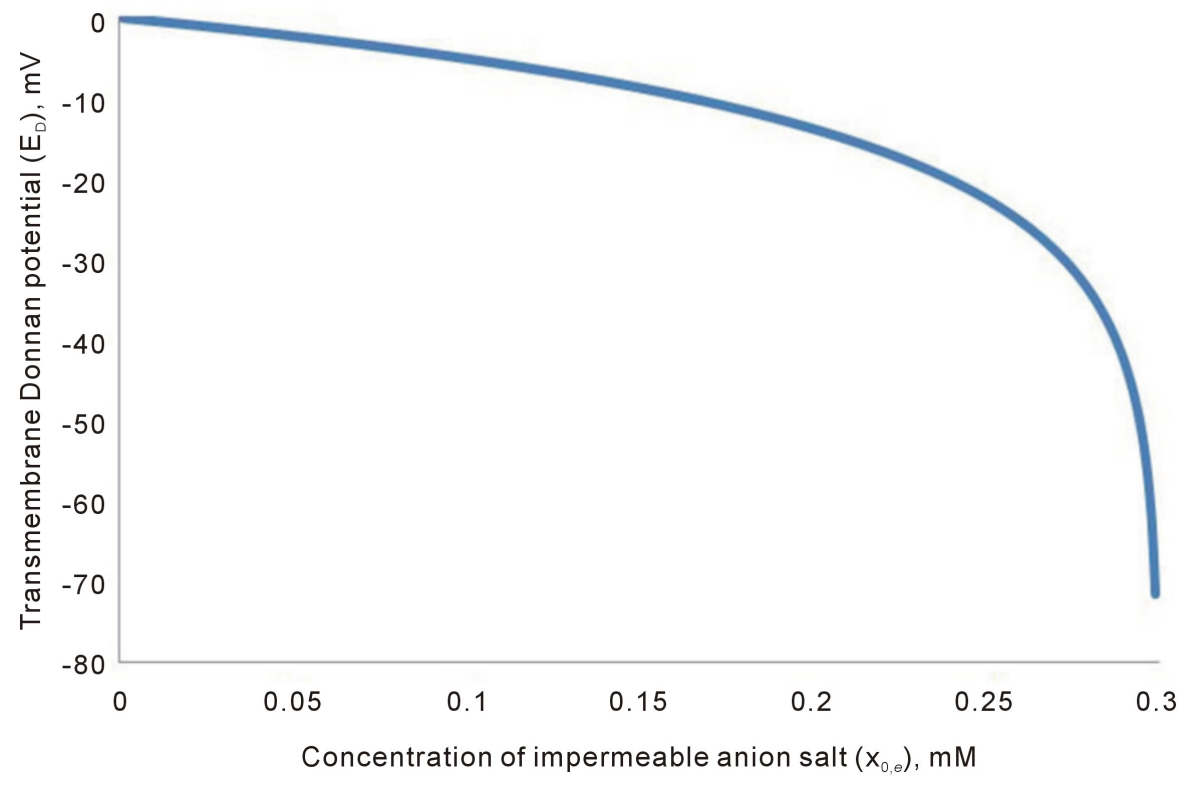

Figure 4. Calculated Donnan potential $\left(E_{D}\right)$ increase in membrane vesicles depending on salt concentration of impermeable anion $\left(x_{0, e}\right)$ initially located in medium. Salts concentrations satisfy such demand $b_{0, i}=b_{0, e}+x_{0, e}=300 \mathrm{mM}$.

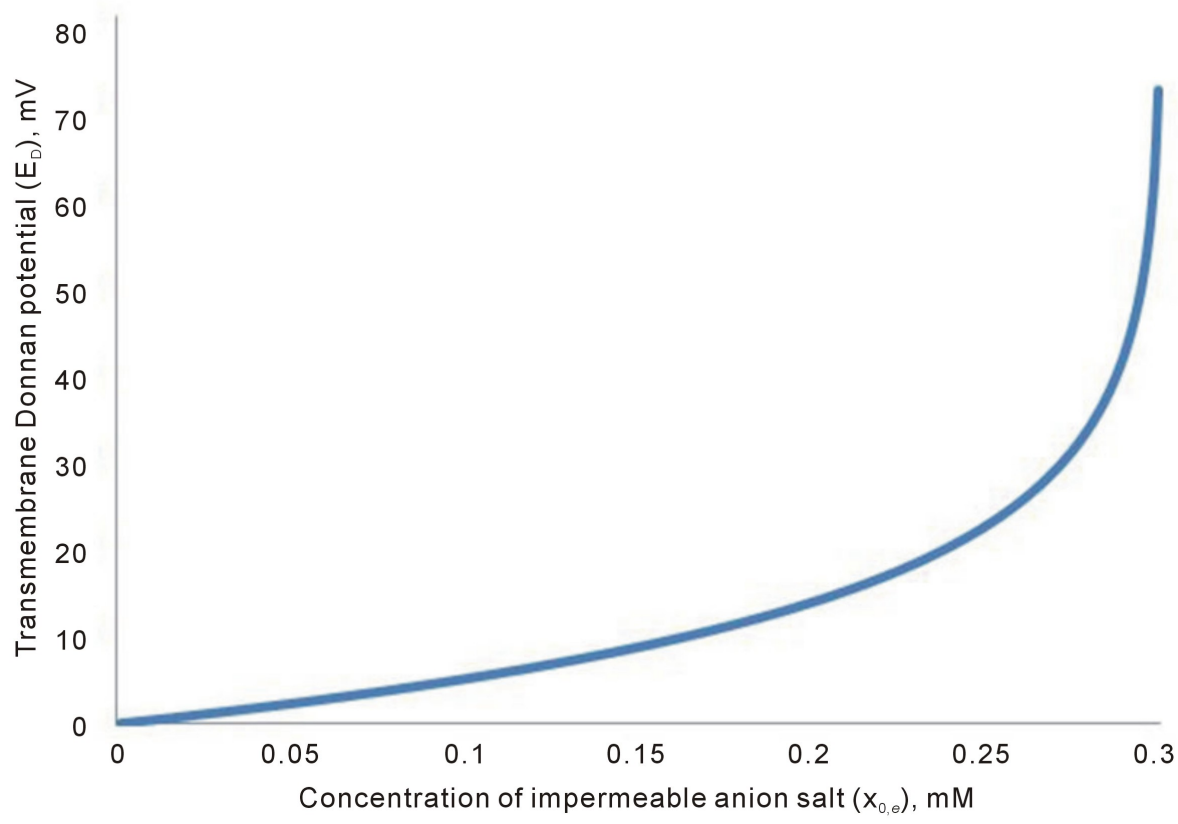

Figure 5. Calculated Donnan potential $\left(E_{D}\right)$ increase in membrane vesicles depending on salt concentration of impermeable cation $\left(z_{0, e}\right)$ initially located in medium. Salts concentrations satisfy such demand $b_{0, i}=b_{0, e}+z_{0, e}=300 \mathrm{mM}$.

trol (2.71) and probe (1.74) experiment, which are statistically different $(n=15, p<0.01)$.

Our results convincingly show that Gibbs-Donnan equilibrium can be used for formation of transmembrane potential in vesicle-like structure. However, the majority of vesicle during preparation is formed as "inside-out" structure and following experiment use vesicle for accumulation of ion, metabolites also of "inside-out" configuration. To simulate transmembrane potential of cell on membrane "inside-out" vesicle potential with negative charge should be created, in other word impermeable anion should be placed in incubation medium. 


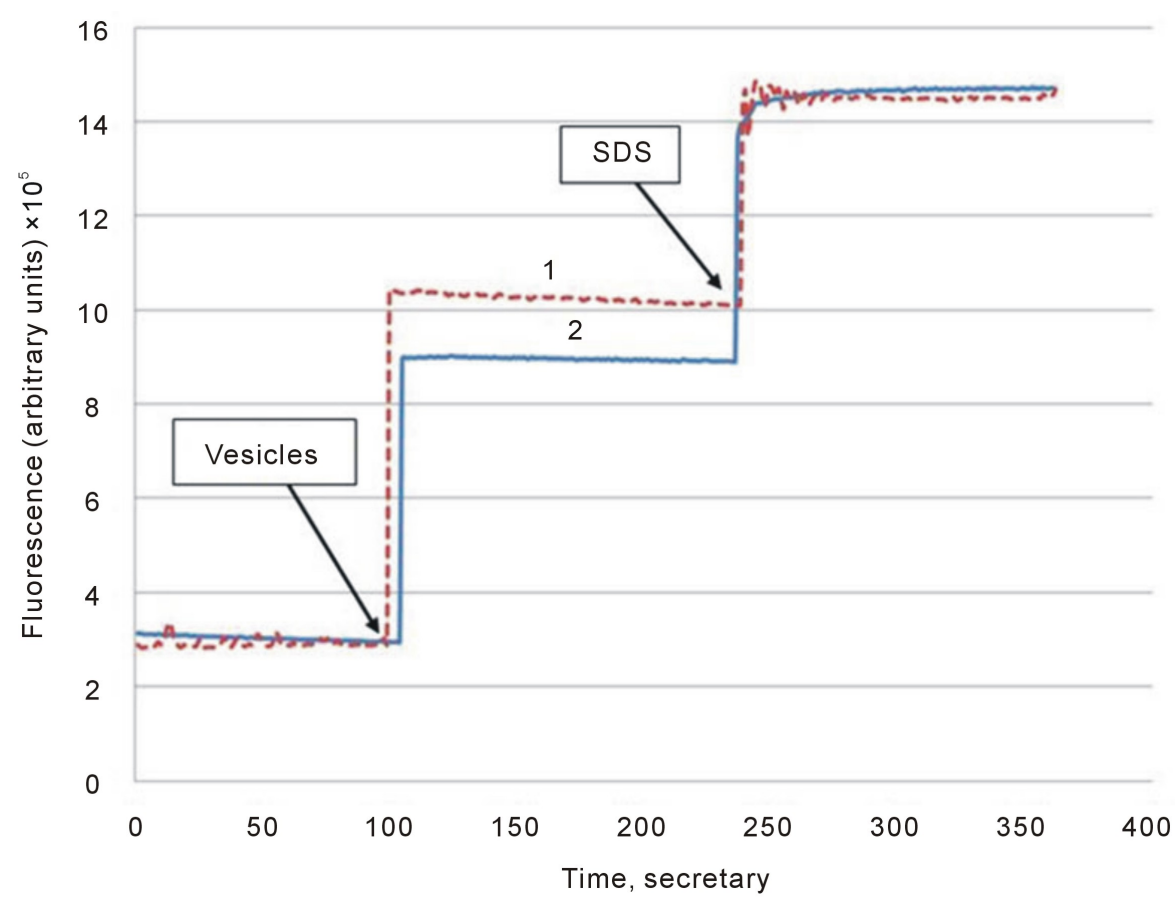

Figure 6. Dependence of fluorescent signal of potential-sensitive dye $\mathrm{DiOC}_{6}(3)$ on plasma membrane vesicles potential: control (1) -medium contained $300 \mathrm{mM} \mathrm{KCl}$, estimated potential is $0 \mathrm{mV}$, probe (2) - medium contain $297 \mathrm{mM}$ cholin chloride and $3 \mathrm{mM} \mathrm{KCl}$, estimated potential is $-65 \mathrm{mV}$. $\mathrm{KCl}$ concentration in vesicle was $300 \mathrm{mM}$. The result of typical experiment is shown.

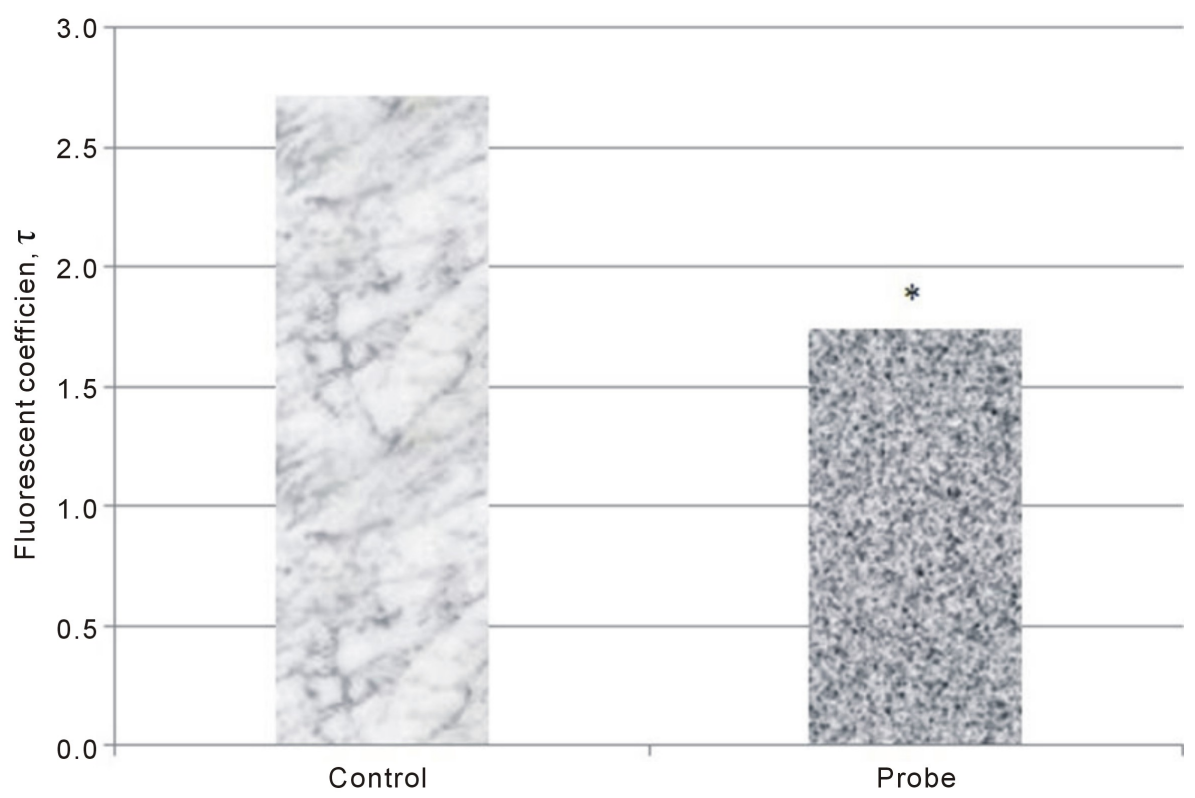

Figure 7. Comparison of fluorescent signal coefficient of potential-sensitive dye $\operatorname{DiOC}_{6}(3)$ for control (medium contain $300 \mathrm{mM} \mathrm{KCl})$ and probe $(297 \mathrm{mM}$ cholin chloride and $3 \mathrm{mM} \mathrm{KCl}$ ) measured on membrane vesicle $(n=15, p<0.01)$.

\subsection{Negative Potential on Vesicle Is Proven by Oxonol VI Spectrum Shift}

According to literature data [32] oxonol VI have red-shift its absorbtion and emission peak when interact with 
liposomes and have a great red-shift if liposomes have inside-positive membrane potential.

In our results, oxonol VI also had red-shifted peak of absorbtion and emission (Figure 8, Figure 9), when vesicle was added, from 590 to 593 in absorption and from 620 to 623 in emission. However great shift from 590 to $602 \pm 1 \mathrm{~nm}$ in absorption and from 620 to $632 \pm 1(\mathrm{n}=6)$ in emission was when vesicle was polarized $(\mathrm{n}=6)$ as a

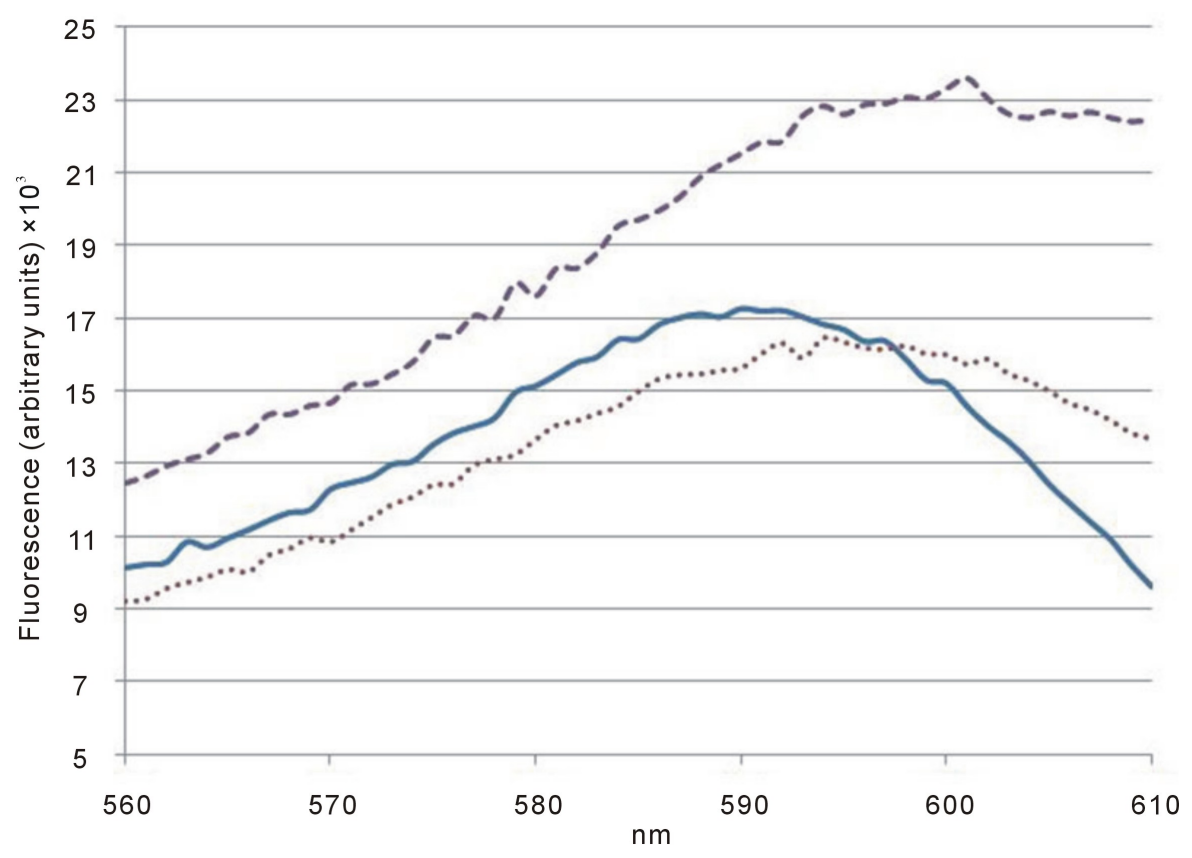

Figure 8. Changing exitation spectrum of oxonol VI after adding membrane vesicles (2) and polarized plasma membrane vesicles (3) comparetively with control in icubation medium (1). The result of typical experiment is shown $(n=6)$.

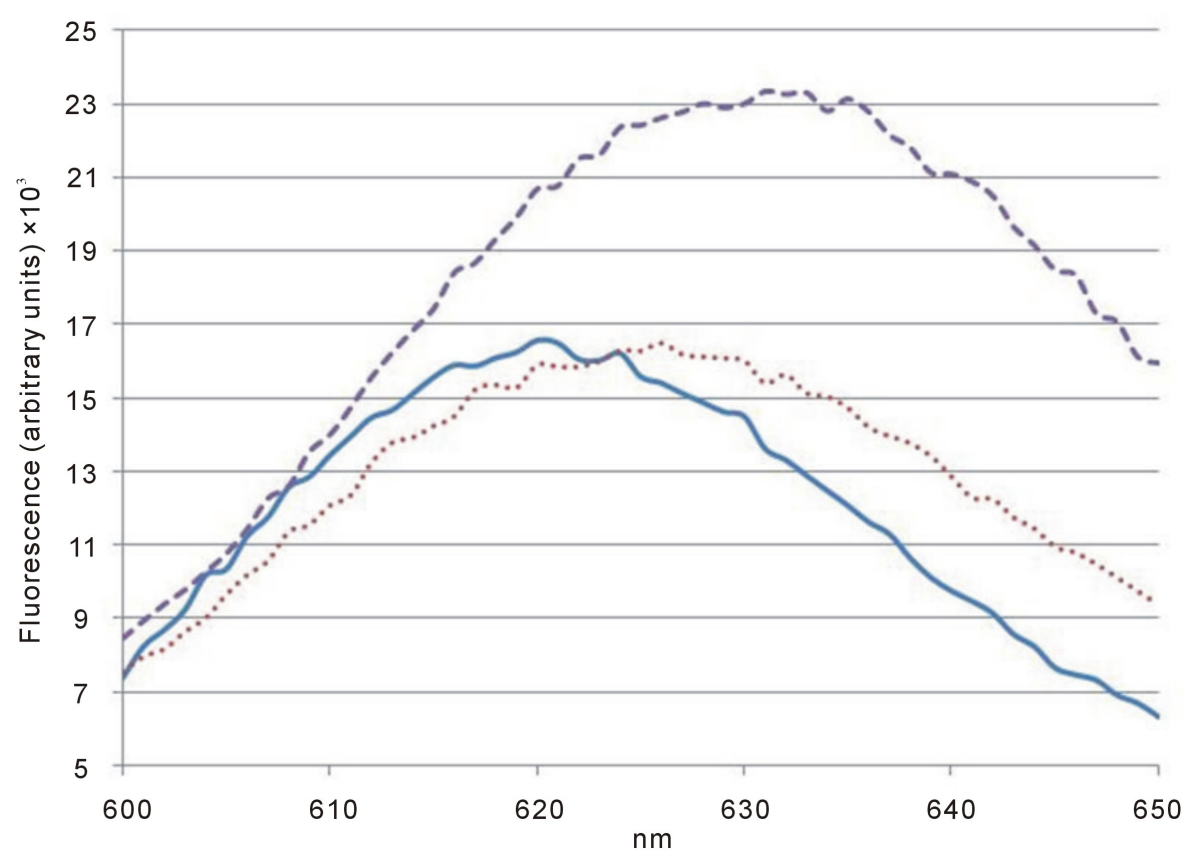

Figure 9. Changing emission spectrum of oxonol VI after adding membrane vesicles (2) and polarized plasma membrane vesicles (3) comparetively with control in icubation medium (1). The result of typical experiment is shown $(n=6)$. 
result of Gibbs-Donnan effect. Control experiment as in previous case was performed in incubation medium which contained $300 \mathrm{mM} \mathrm{KCl}$ and $200 \mu \mathrm{g}$ vesicles, which contained also $300 \mathrm{mM} \mathrm{KCl}$. Sample probe contained 297 potassium citrate (as impermeable anion) and $3 \mathrm{mM} \mathrm{KCl}$ with vesicle of the same composition.

\section{Conclusions}

The influence of Donnan effect on membrane polarization when size of one compartment is very different from another has been reviewed in this research.

It should be emphasized that location of impermeable ion influences on the value of transmembrane potential that will be obtained. Impermeable ions location in incubation medium creates transmembrane potential 6 times as high as location of corresponding ions in membrane vesicles. It can be explained by the different particles number. However, salt concentration is equal but compartment volumes are significantly different, that means various numbers of available particles and load limit in each compartment. Concentration gradient directed out from the smaller compartment is better for higher polarization, as its load limit in equilibrium point is relatively low.

Therefore, location of impermeable ion in incubation medium creates stable Donnan potential which depends on 1) concentration of ion: the higher concentration is the higher membrane potential can be created; 2) charge of ion: impermeable anion creates a negative transmembrane potential and cation forms a positive transmembrane potential. Different sign of polarization can be used for studying depolarization-hyperpolarization event during cell excitation.

Theoretical calculations were confirmed via using two different potential-sensitive probes in diverse incubation medium. It was expected that when impermeable cation (in this case choline) located in medium and vesicle contain equivalent concentration of permeable cation (potassium) positive transmembrane potential should be formed. This was confirmed by different fluorescence quantum yield of potential-sensitive probe $\mathrm{DiOC}_{6}(3)$. In other case oxonol VI was used for verifying vesicle polarization where impermeable anion (citrate) placed in medium and vesicle was fulled by tantamount concentration of permeable anion (chloride). The shift of spectrum of specified probe in emission and excitation spectrum was noted in this case and according to Apell [32] testifies membrane vesicle polarization.

Thereby, Gibbs-Donnan effect can be used in vesicular method of investigation in order to form stable transmembrane potential. In further investigation stable transmembrane potential in membrane vesicles can be used for analysis electrical-sensitivity of transmembrane ion transporters, for example, the plasma membrane $\mathrm{Mg}^{2+}$, ATP-dependent $\mathrm{Ca}^{2+}$-pump.

\section{References}

[1] Sunose, H., Ikeda, K., Saito, Y., Nishiyama, A. and Takasaka, T. (1992) Membrane Potential Measurement in Isolated Outer Hair Cells of the Guinea Pig Cochlea Using Conventional Microelectrodes. Hearing Research, 62, 237-244. http://dx.doi.org/10.1016/0378-5955(92)90190-X

[2] Berggren, P. and Sohtell, M. (1986) Microelectrode Studies of D-Glucose ${ }^{-}$and $\mathrm{K}^{+}$-Induced Changes in Membrane Potential of Electrofused Insulin-Producing Cells. FEBS Letters, 202, 367-372. http://dx.doi.org/10.1016/0014-5793(86)80720-7

[3] Nobes, C.D. and Brand, M.D. (1989) A Quantitative Assessment of the Use of ${ }^{36} \mathrm{Cl}^{-}$Distribution to Measure Plasma Membrane Potential in Isolated Hepatocytes. BBA—Biomembranes, 987, 115-123.

[4] Cuevas, J. (2007) The Resting Membrane Potential. In: Enna, S.J., Bylund, D.B., Eds., xPharm: The Comprehensive Pharmacology Reference, Elsevier Inc., New York, 1-4.

[5] Hodgkin, A.L. and Huxley, A.F. (1945) Resting and Action Potentials in Single Nerve Fibres. The Journal of Physiology, 104, 176-195.

[6] Andersen, S.S.L., Jackson, A.D. and Heimburg, T. (2009) Towards a Thermodynamic Theory of Nerve Pulse Propagation. Progress in Neurobiology, 88, 104-113. http://dx.doi.org/10.1016/j.pneurobio.2009.03.002

[7] Guiet-Bara, A., Ibrahim, B., Leveteau, J. and Bara, M. (1990) Calcium Channels, Potassium Channels and Membrane Potential of Smooth Muscle Cells of Human Allantochorial Placental Vessels. Bioelectrochem. Bioenergetics, 48, 407-413. http://dx.doi.org/10.1016/S0302-4598(99)00020-3

[8] Vamosi, G. (2006) The Role of Supramolecular Protein Complexes and Membrane Potential in Transmembrane Signaling Processes of Lymphocytes. Immunology Letters, 104, 53-58. http://dx.doi.org/10.1016/j.imlet.2005.11.014 
[9] Chimerel, C., Field, C.M., Piñero-Fernandez, S., Keyser, U.F. and Summers, D.K. (2012) Indole Prevents Escherichia coli Cell Division by Modulating Membrane Potential. BBA-Biomembrane, 1818, 1590-1594.

[10] Goudeau, H., Goudeau, M. and Guibourt, N. (1992) The Fertilization Potential and Associated Membrane Potential Oscillations during the Resumption of Meiosis in the Egg of the Ascidian Phallusia mammillata. Developmental Biology, 153, 227-241. http://dx.doi.org/10.1016/0012-1606(92)90108-S

[11] Khelashvili, G., Weinstein, H. and Harries, D. (2008) Protein Diffusion on Charged Membranes: A Dynamic Mean-Field Model Describes Time Evolution and Lipid Reorganization. Biophysical Journal, 94, 2580-2597. http://dx.doi.org/10.1529/biophysj.107.120667

[12] Strahl, H. and Hamoen, L.W. (2010) Membrane Potential Is Important for Bacterial Cell Division. Proceedings of the National Academy of Sciences of the United States of America, 107, 12281-12286. http://dx.doi.org/10.1073/pnas.1005485107

[13] Kralj, J.M., Hochbaum, D.R., Douglass, A.D. and Cohen, A.E. (2011) Electrical Spiking in Escherichia coli Probed with a Fluorescent Voltage-Indicating Protein. Science, 333, 345-348. http://dx.doi.org/10.1126/science.1204763

[14] Hell, J.W. and Jahn, R. (1998) Bioenergetic Characterization of $\gamma$-Aminobutyric Acid Transporter of Synaptic Vesicles. Methods in Enzymology, 296, 116-124. http://dx.doi.org/10.1016/S0076-6879(98)96010-4

[15] Dudeja, P.K., Tyagi, S., Kavilaveettil, R.J., Gill, R. and Said, H.M. (2001) Mechanism of Thiamine Uptake by Human Jejunal Brush-Border Membrane Vesicles. American Journal of Physiology-Cell Physiology, 281, 786-792.

[16] Krick, W., Wolff, N.A. and Burckhardt, G. (2000) Voltage-Driven p-Aminohippurate, Chloride, and Urate Transport in Porcine Renal Brush-Border Membrane Vesicles. Pflügers Archiv, 441, 125-132. http://dx.doi.org/10.1007/s004240000378

[17] Malo, C. and Wilson, J.X. (2000) Glucose Modulates Vitamin C Transport in Adult Human Small Intestinal Brush Border Membrane Vesicles. Journal of Nutrition, 130, 63-69.

[18] Zelikovic, I. and Budreau-Patters, A. (1999) $\mathrm{Cl}^{-}$and Membrane Potential Dependence of Amino Acid Transport across the Rat Renal Brush Border Membrane. Molecular Genetics and Metabolism, 67, 236-247. http://dx.doi.org/10.1006/mgme.1999.2866

[19] Freel, R.W., Hatch, M. and Vaziri, N.D. (1998) Conductive Pathways for Chloride and Oxalate in Rabbit Ileal Brush-Border Membrane Vesicles. American Journal of Physiology, 275, 748-757.

[20] Krick, W., Dölle, A., Hagos, Y. and Burckhardt, G. (1998) Characterization of the Chloride Conductance in Porcine Renal Brush-Border Membrane Vesicles. Pflügers Archiv, 435, 415-421. http://dx.doi.org/10.1007/s004240050532

[21] Yanagawa, N., Jo, O.D. and Said, H.M. (1997) Riboflavin Transport by Rabbit Renal Brush Border Membrane Vesicles. Biochimica et Biophysica Acta, 1330, 172-178. http://dx.doi.org/10.1016/S0005-2736(97)00153-3

[22] Bönisch, H. (1998) Transport and Drug Binding Kinetics in Membrane Vesicle Preparation. Methods in Enzymology, 296, 259-278. http://dx.doi.org/10.1016/S0076-6879(98)96020-7

[23] Garaiová, Z., Mohsin, M., Vargová, V., Banica, F.-G. and Hianik, T. (2012) Complexation of Cytochrome c with Calixarenes Incorporated into the Lipid Vesicles and Supported Membranes. Bioelectrochemistry, 87, 220-225. http://dx.doi.org/10.1016/j.bioelechem.2011.12.004

[24] Barts, P.W.J.A. and Borst-Pauwels, G.W.F.H. (1985) Effects of Membrane Potential and Surface Potential on the Kinetics of Solute Transport. Biochimica et Biophysica Acta (BBA)—Biomembranes, 813, 51-60. http://dx.doi.org/10.1016/0005-2736(85)90344-X

[25] Zhan, H. and Lazaridis, T. (2012) Influence of the Membrane Dipole Potential on Peptide Binding to Lipid Bilayers. Biophysical Chemistry, 161, 1-7. http://dx.doi.org/10.1016/j.bpc.2011.10.002

[26] Babich, L.G., Fomin, V.P. and Kosterin, S.A. (1990) Effect of the Membrane Potential on the $\mathrm{Mg}^{2+}$,ATP-Dependent Transport of $\mathrm{Ca}^{2+}$ across Smooth Muscle Sarcolemma. Biohymia (in Russian), 55, 1890-1901.

[27] Veklich, T.O. and Kosterin, S.A. (2005) Comparison Analysis of Myometrium Plasma Membrane $\mathrm{Na}^{+}$, $\mathrm{K}^{+}$-ATPase and $\mathrm{Mg}^{2+}$-ATPase Properties. Ukrain Biochemical Journal (in Ukrainian), 77, 66-75.

[28] Bradford, M.M. (1976) A Rapid and Sensitive Method for the Quantitation of Microgram Quantities of Protein Utilizing the Prinsiple of Protein-Dye Binding. Analytical Biochemistry, 72, 248-282. http://dx.doi.org/10.1016/0003-2697(76)90527-3

[29] Sperelakis, N. (2012) Gibbs-Donnan Equilibrium Potentials. In: Sperelakis, N., Ed., Cell Physiology Source Book, Cell Physiology Source Book, Inc., San-Diego, 147-151.

[30] Kosterins, S.A. and Cherny, A.P. (1991) Gibbs-Donnan Equilibrium in the System Membrane Vesicles-Incubation Medium. Biophysica (in Russian), 36, 826-829.

[31] Hoffman, J.F. and Laris, P.C. (1974) Determination of Membrane Potentials in Human and Amphiuma Red Blood 
Cells by Means of a Fluorescent Probe. The Journal of Physiology, 239, 519-552.

[32] Apell, H.-J. and Bersch, B. (1987) Oxonol VI as an Optical Indicator for Membrane Potentials in Lipid Vesicles. BBA-Biomembranes, 903, 480-494. 